\title{
Capacidades metacognitivas en el sistema educativo en instituciones educativas de educación media
}

\section{Metacognitive capacities in the educational system in educational institutions of secondary education}

\author{
Natalie del Carmen Muñoz Morales \\ natalie.munoz@profe.umcervantes.cl \\ Universidad Miguel de Cervantes \\ Chile \\ https://orcid.org/0000-0002-7079-3099 \\ Nicolás Pablo Barrientos Oradini \\ nbarrientoso@docente.uss.cl \\ Universidad San Sebastián \\ Chile \\ https://orcid.org/0000-0002-5854-9501
}

Luis Araya Castillo

luis.arayac@uautonoma.cl

Universidad Autónoma de Chile

Chile

https://orcid.org/0000-0001-7574-3907

Jaime Reyes Saavedra

ean.umc@gmail.com

Universidad Miguel de Cervantes

Chile

Recibido: 05 de noviembre del 2018

Aprobado: 10 de diciembre del 2018

\section{RESUMEN}

El propósito del presente artículo es analizar las habilidades metacognitivas en estudiantes de 5to medio en Santiago de Chile. La investigación se sustentó en los postulados teóricos de Caballero (2010), Woolfolk (2006), Castejón (2009), Crozier (2001), (2012), Hernán (2014), entre otros. El estudio se enmarcó como descriptivo, con un diseño de campo, no experimental y transeccional. La población estuvo conformada por un total de ciento diecinueve (119) sujetos, estudiantes de quinto (5to) medio de las Instituciones objeto de estudio. Los resultados mostraron que los estudiantes consideran altamente adecuado las habilidades de evaluación, así como la orientación 
a la acción frente a la volatilidad como habilidad metacognitiva. Se concluyó que las habilidades metacognitivas son altamente adecuadas en opinión de los estudiantes, por lo que se recomendó fortalecer la capacidad metacognitiva de los estudiantes mediante del incentivo al uso de dispositivos en el proceso enseñanza aprendizaje.

Descriptores: Aprendizaje activo; Aprendizaje a través de la experiencia; habilidad pedagógica; evaluación del docente; proceso cognitivo.

\section{ABSTRACT}

The purpose of this article is to show the results of the research was aimed to analyze the development of metacognitive skills in students 5th grade of the Industrial Technical Educational The purpose of this article is to analyze metacognitive skills in 5th grade students. The research was based on the theoretical postulates of Caballero (2010), Woolfolk (2006), Castejón (2009), Crozier (2001), (2012), Hernán (2014), among others. The study was framed as descriptive, with a field design, not experimental and transeccional. The population consisted of a total of one hundred nineteen (119) subjects, students of the fifth (5th) average of the institutions under study. The results of the results of the results. It was concluded that the metacognitive skills are the ones that improve the opinion of the students, so it is recommended to strengthen the metacognitive capacity of the students through the use of devices in the teachinglearning process.

Descriptors: Active learning; Learning through experience; pedagogical ability; teacher evaluation; cognitive process.

\section{INTRODUCCIÓN}

El ser humano se ha caracterizado por la búsqueda de formas diferentes de aprendizajes orientados a desarrollar conocimientos en su proceso evolutivo, cambiar actitudes y formas de resolver problemas, hasta llegar al punto de convertir este proceso en una condición permanente a lo largo de toda la vida. Con el transcurrir del tiempo, la tecnología se ha hecho imperativa en esa búsqueda y los seres humanos han volcado sus esfuerzos hacia su uso y apropiación en los diferentes espacios.

Crozier (2001), sostiene que es conveniente modificar de manera constante las estrategias, los modelos, los ejemplos, tanto la teoría como la práctica del discurso, las formas, reglas así como también las normas de comportamiento, pensamiento y desenvolvimiento de la vida en sus diversas manifestaciones, convirtiéndose al mismo 
tiempo en un reto para la adquisición de habilidades y estrategias de pensamiento que permitan alcanzar el conocimiento.

En este orden de ideas en el estudiante se debe impartir el conocimiento de tipo cognitivo, praxeológico y actitudinal, el primero relacionado con lo teórico relacionado con el saber qué y el saber por qué, el segundo está orientado a la acción, al saber cómo y construcción de conocimiento, el tercero con el ser, con las actitudes mismas del sujeto con relación al aprendizaje y con el factor motivacional razones muy importante debido que si esto siguen continúan se va a incrementar o aumentar la brecha de calidad educativa, una disminución en la conclusión escolar y estudiantes cada vez menos competentes. (Necuzzi, 2013).

Es significativo entonces, determinar que el enfoque metacognitivo es derivado de las estudios de las áreas de la psicología cognitiva. Se refiere al grado de conciencia o conocimiento que las personas tienen como manera de pensar que implican procesos y eventos cognitivos, así como las estructuras de contenidos y la habilidad para ejercer control de dichos procesos a fin de organizarlos, revisarlos y modificarlos en función de los resultados del aprendizaje. (Chadwick citado en Blanco, 2015).

En las capacidades metacognitivas, menciona Hernán (2014), la tecnología provee diversidad de recursos para el logro de estas, como son: aulas virtuales, salones con video-enlaces, conexión a la red mundial y dispositivos móviles, también conocido como m-learning, que se interpreta como la concurrencia del aprendizaje electrónico con dispositivos móviles, generalmente en un marco de referencia educativo diferente al de un salón de clase y un profesor.

En relación con lo antes expuesto en el contexto mundial, Europa se ha consolidado como la región líder en cuanto a la tasa de penetración de la telefonía móvil, muy por encima de otras regiones, en algunos países de este continente, España tiene una tasa de penetración del $144 \%$ según datos de la Unión Internaciones de Telecomunicaciones (UIT, 2009).

De acuerdo con lo planteado por Rodríguez (citado en Lizardo, 2014), el aprendizaje móvil busca ser la tecnología educativa del nuevo siglo, basándose en una instrucción 
formal e informal en cualquier momento o espacio geográfico dando la oportunidad de controlar el tiempo y aprovecharlo para el aprendizaje, llegando a tocar diferentes niveles y lugares en el mundo.

Es importante hacer énfasis que la creciente incorporación de los dispositivos móviles en el mundo, confirma lo que pudiese ser una necesidad social imperante por estar permanentemente comunicado con el entorno, especialmente en el caso de las nuevas generaciones o los llamados nativos digitales, ya que un estudio reciente realizado por la UNESCO (2013), señala que los jóvenes utilizan los móviles sin ninguna orientación por parte de los acudientes y en el peor de los casos sin el adiestramiento u orientación de las Instituciones educativas, es ahí donde empieza los usos indebidos de este tipo de tecnologías.

Es preciso decir que en América del Norte gran parte de la población estudiantil emplea la tecnología de información y comunicación más como objeto de distracción y ocio que para fines educativos, los educando no implementan tácticas de estudio apoyadas en estas herramientas, causa de ello es por el poco aprestamiento que le han suministrado las organizaciones educativas, no se les ha brindado la oportunidad de desarrollas las capacidades cognitivas con la utilización de los dispositivos móviles (Necuzzi, 2013).

Paralelamente en Centroamérica precisamente en México, Hernán (2014), refiere que el $84 \%$ de los estudiantes utilizan celulares o móviles en diversas actividades escolar, estas son descritas según con el instrumento con los siguientes resultados el $82 \%$ para llamar, para jugar un 19\%, para sacar fotos un 33\%, el $45 \%$ escriben mensajes, un $19 \%$ escucha música, para ver videos un $7 \%$ y el Bluetooth $13 \%$.

Lo anteriormente mencionado, condujo al investigador a inferir a que el estudiante se les debe desarrollar actividades didácticas con enfoque mixto en el aprendizaje para mejorar México falta de atención, capacidad mental, maduración emotividad e interés que presentan los estudiantes.

En este orden de ideas, América Latina afronta relevantes desafíos en los procesos educativos, entre ellos están: programas de capacitación del cuerpo docente 
insuficientes, analfabetismo en adultos, altas tasas de deserción especialmente en la educación secundaria, limitado acceso a la educación, particularmente a la educación inicial y preescolar; así lo plantea la (UNESCO, 2013). Cada uno de los aspectos mencionados anteriormente son causantes de la baja calidad educativa.

Es preciso destacar que estas problemáticas se manifiestan particularmente en poblaciones de bajos recursos urbanos, rurales y en poblaciones indígenas, recientemente, actores educativos del sector público como privado han comenzado a explorar el aprendizaje móvil para enfrentar de las necesidades educativas más prioritarias de la región. (UNESCO, 2012).

De acuerdo con lo anterior, y en continuidad a los señalamientos de la UNESCO (2012), en América Latina y el Caribe mientras el uso de teléfonos móviles en la sociedad está aumentando ágilmente, las iniciativas de incorporar el aprendizaje móvil se encuentran aún en niveles iniciales de desarrollo, la mayoría de los programas son proyectos piloto, impulsados por organizaciones sin fines de lucro o universidades, que típicamente se dirigen a pequeños grupos y focalizan en necesidades particulares 0 locales.

A nivel de las políticas, es observado el aprendizaje móvil un auge en todos los ámbitos, sin embargo, existen limitaciones con respecto a utilizar los dispositivos móviles dentro del aula de clase, específicamente los teléfonos celulares, tales normativas son impuestas por entes gubernamentales o instituciones que se dedican a regular la educación, pero tal situación está tornándose menos estricta en algunos países.

Por lo tanto, la calidad en los procesos de aprendizaje de la educación que la mayoría de los estudiantes obtienen en América Latina se encuentra en un nivel bajo de acuerdo con (Castejon, 2009), quien además indica que en las instituciones oficiales donde se encuentra en gran proporción los niños y niñas de básica primaria, secundaria y virtualmente todos los sectores son de estratos sociales bajos. Donde las metas están encaminadas en la cobertura, disminuyendo la atención en los procesos y los 
resultados del aprendizaje. Es decir que no se ha logrado desarrollar la transformación del sistema educativo para construir escenarios de igualación de oportunidades.

En ese sentido, Caballero (2010), afirma que los docentes logran describir de forma moderada las teorías de aprendizaje que se identifican con el uso de las tecnologías móviles en el desempeño escolar, dominan los aspectos positivos e identifican los tipos de tecnologías, sin embargo se convierte en un desafío para poder modificar y perfeccionar día tras días las diferentes formas de contribuir con los procesos de enseñanza sin el respaldo de políticas educativas establecidas y estructuradas, en la búsqueda de fortalecer el quehacer pedagógico.

De allí que se pretenda analizar cómo se presentan las habilidades metacognitivas en estudiantes de 5to medio en instituciones de educación media en la región metropolitana de la ciudad de Santiago en Chile, para ampliar el conocimiento sobre la posible influencia del uso de las tecnologías de información en el desarrollo de éstas capacidades. La selección de la población estuvo orientada en seleccionar intencional de estudiantes activos y cursante del 5 to grado de las instituciones educativas.

\section{DESARROLLO}

A los efectos de una mayor inteligencia del presente artículo, se desarrolló un esquema de trabajo, que constituye su sustentación teórica y que se estructuró de la manera que de seguida se ofrece:

\section{Habilidades metacognitivas}

Es relevante abordar lo planteado por Woolfolk (2006), quién define la metacognición como la conciencia que tiene la gente sobre su propia maquinaria cognoscitiva y sobre la forma en que funciona ésta. Así mismo señala que literalmente significa "conocimiento sobre los conocimientos y el aprendizaje" (pag,54), el cual es utilizado tanto para monitorear como regular los procesos cognoscitivos como el razonamiento, la comprensión, la solución de problemas, el aprendizaje, entre otros. 
A su vez implica elegir la mejor forma para manejar tarea de aprendizaje como lo hacen los estudiantes que tienen altas habilidades metacognitivas que organizan sus actividades, seleccionando entre varios para aprender y modifican sus estrategias si es necesario.

Por su parte Chadwick (1985) citado por Blanco (2015), señala que las habilidades metacognitivas pueden considerarse como el nivel conocimiento que desarrollan los individuos sobre sus formas de pensar, es decir, sus procesos cognitivos, los contenidos y la habilidad orientada a controlar dichos procesos con la finalidad de organizarlos, revisarlos y modificarlos en función de los progresos, así como de los resultados del aprendizaje. Estas habilidades mejoran de forma importante durante las edades de niñez, tanto en lo que concierne a la memoria como en lo referente a otros ámbitos como la atención o la lectura.

Necuzzi, (2013), lo explica como la consciencia que tiene el estudiante de habilidades concretas como orientación, establecimiento de un plan de acción, monitorización dejando a un lado sus propios métodos de estudio logrando sus objetivos de aprendizaje, y un alto rendimiento en sus exámenes o controles. Del mismo modo el autor advierte que el éxito de las mismas depende crucialmente de los esfuerzos del profesor para ayudar a interiorizarlas en los alumnos y de esa forma evitar efectos que tengan relación con el desgaste emocional en los estudiantes y que conlleve a afectar el rendimiento académico (Caballero, Abello, y Palacio, 2007).

En referencia a lo antes planteado el autor refleja coincidencia en que las estrategias que promuevan las habilidades cognitivas cuentan con un uso limitado si los estudiantes no están motivados para aprovecharlas haciendo hincapié que la adquisición de las habilidades cognitivas es un proceso de largo plazo y que implica innumerables horas de prácticas para aprender a autorregularlas en el proceso de establecimiento de objetivos. 


\section{Planeación}

Según Woolfolk (2006), la planeación implica cuanto tiempo dedicar a una tarea, que estrategias utilizar, como empezar, que recursos reunir, que orden seguir, que revisar de forma superficial y a que ponerle mayor atención. Quiere decir entonces que planificar implica anticiparse a las acciones que han de tomarse con el fin de lograr el aprendizaje significativo, lo cual forma constituye decisiones y acciones realizadas de forma sistemática además de ordenada constituyen lo que se denomina el proceso de planeación.

En palabras de Chadwick (1985) citado por Blanco (2015), es organizar los métodos o recursos a utilizar conforme a un plan y plan es una determinada idea. La planeación exige conocer todos los sucesos, todos los recursos necesarios, todos los medios comprometidos, todos los modelos, todos los procesos, todas las posibilidades de variabilidad, todas las capacidades; sin embargo, como no es posible saber todo lo que se está haciendo, la planeación estará limitada por todo lo especificado y fundamentalmente por quien la realiza.

Por su parte Flavell, (1978), explica que la planeación no solo es una función administrativa que ayuda a señalar objetivos o a programar objetivos, sino es un criterio de vida que permite tener una perspectiva temporal de largo plazo para las actividades y metas. De la misma manera implica un conocimiento o contacto personal con el núcleo más íntimo del estudiante o persona que incluye la conjunción del pensamiento, las emociones, los valores, el cuerpo y las experiencias vitales que dan origen a sí mismo, es decir, al yo personal que solo puede ser captado en un proceso intuitivo que transciende a la razón.

En relación a las definiciones antes planteadas los diferentes autores coinciden en que se debe estar consciente de los recursos o herramientas a utilizar en el proceso de aprendizaje, sin embargo Casares y Siliceo (2014), va un poco más allá especificando que se trata de un contacto con el interior de la persona para de esta manera posee una visión a largo plazo de sus metas u objetivos preestablecidos. 
El criterio de Woolfolk (2006) permite entender el proceso de planeación como el tiempo, recursos, pasos, atención que conlleva las metas a alcanzar, permitiéndole al individuo conocer las actividades a ejecutar en el proceso de enseñanza-aprendizaje lo cual le permitiría una mayor organización por ende un mayor rendimiento en todo lo que se proponga en su vida cotidiana o escolar. En palabras de Cabrera y Galán (2002), ello fortalecería los momentos de satisfacción académica en los estudiantes, permitiendo no sólo lograr los objetivos y metas escolares sino potenciar el rendimiento cognitivo mejorando de ésta manera las capacidades desarrolladas.

\section{Verificación}

Siguiendo la argumentación de Woolfolk (2006), la verificación es la conciencia continua de preguntarse la manera en que se están haciendo las cosas, de tal manera que verificar en indagar si tiene sentido lo ejecutado, si se ha tomado el tiempo suficiente para realizarlo o si se ha estudiado lo necesario, es decir que es reafirmar que se está haciendo lo correcto, o reconstruir lo planeado para hacerlo de la manera correcta que le permita al estudiante concretar lo propuesto como pasar el examen con una buena calificación, realizar proyectos de investigación, asegurar su futuro de un modo correcto, entre otros.

Por su parte, Necuzzi, C (2013), considera que es la etapa más importante pues permite el análisis y la comprensión de lo que está sucediendo lo cual si se está logrando de forma correcta continuarlo, sino redireccionarlo para conseguir el fin deseado. Convirtiéndose en una excelente oportunidad de autodescubrimiento en diferentes dimensiones, así como también en una retroalimentación, la cual finaliza con éxito siempre y cuando el estudiante sea consciente de cuáles son las variables que condicionan sus resultados.

Por otra parte Castejón, J (2009), explica que aun cuando el paso anterior del proceso en la implementación de un plan en este caso de las habilidades cognitivas se realice de manera correcta, nada garantiza que el plan resultará de manera exitosa. Por lo tanto se debe verificar las acciones a seguir preguntándose ¿Se están llevando a cabo 
acciones necesarias en el tiempo correcto? ¿Se están haciendo con el nivel de calidad que se desea y se requiere? Entre otras preguntas, con el fin de asegurarse que este siendo desarrollado de manera efectiva brindado mejores resultados.

Partiendo de los conceptos basados en los autores precitados cada uno de ellos coinciden que la verificación consiste en corroborar las acciones que se están realizando para conocer si se están realizando de una manera correcta, pero Necuzzi, C (2013), es un poco más específico señalando que es una etapa que toma relevancia al momento de obtener resultados positivos puesto que es oportuna para enmendar los errores que se están provocando o provocarían.

Sin embargo, Woolfolk (2006), la define como reafirmar pero a su vez asegurar gran parte del éxito de la implementación del plan de estudio, cuestionándose de manera constante ya que posee factores críticos u objetivos específicos que marcan el progreso a lo largo de la trayectoria completa anticipándose a situaciones que puedan interrumpir el mismo.

\section{Evaluación}

En palabras de Unesco (2012), la define como el monitoreo de los resultados, si los objetivos se definieron bien desde el comienzo del proceso, si se obtuvo el desempeño esperado, consecuencias inesperadas positivas o negativas las cuales se convierten en fuente valiosa de aprendizaje, si se realización la preparación adecuada, en otras palabras la evaluación es un proceso donde se emiten juicios basados en la información obtenida luego de emitidos los pasos o actividades realizadas.

Por su parte Woolfolk (2006), señala que consiste en hacer juicios acerca de los procesos y resultados tanto del pensamiento como del aprendizaje emitiéndose preguntas como sobre las estrategias implementadas, las mejoras a lo realizado, la continuidad del proyecto, el grado de satisfacción una vez culminado el modelo, la tarea, el plan, entre otros, para de esta manera evitar la intervención de los razonamientos espontáneos incorrectos en el aprendizaje. 
Necuzzi, (2013), la define como el juicio explicitado o no de sus actividades cognitivas o del producto mental de las mismas. Para que de esta manera el individuo tome decisiones para modificar o no sus actividades cognitivas así como también sus productos, pero a su vez otros aspectos de la situación de aprendizaje en función al juicio metacognitivo, identificando dificultades que sesgan sus habilidades metacognitivas, pudiendo proponer acciones pedagógicas a implementar para ayudar a superarlas.

Los autores precitados coinciden en que la evaluación es un juicio en base a los resultados obtenidos en el proceso de aprendizaje metacognitivo. Particularmente Woolfolk (2006), señala algunas preguntas que para él son necesarias luego de concluido el proceso para la obtención de la información.

Define evaluación como el juicio de los resultados que emitió el desarrollo del proceso metacognitivo, lo cual permite obtener información a partir de una serie de preguntas para de esta manera activar un proceso mental sobre las actividades cognitivas realizadas generando decisiones que modifiquen los productos negativos ocasionados permitiéndole al estudiante mejorar en futuros planes.

Por otra parte Necuzzi, (2013), hacen mención a lo relevante que es tomar atención ante las causas que producen la inadecuación de los estudiantes ante una determinada situación para de esta manera propiciarles solución.

\section{Diferencias individuales de la metacognición}

Según Woolfolk (2006), significan el resultado del desarrollo, debido a que el individuo crece volviéndose capaz de ejercitar el control ejecutivo sobre las estrategias, determinando si ha entendido las instrucciones o si estudio lo suficiente para recordar un conjunto de conceptos. No todas las diferencias están relacionadas con la edad o maduración sino que existe una gran variabilidad incluso en estudiantes con el mismo nivel de desarrollo, y que posiblemente se originan por diferencias biológicas o por algunas variaciones en las experiencias de aprendizaje. 
Mientras que Castejón (2009), las define como las dificultades o problemas de aprendizaje que en a veces se asocian a ciertas expectativas, motivaciones, estilos de aprendizaje y otros factores de personalidad, que constituyen uno de los elementos a tener en cuenta al iniciar el proceso de enseñanza-aprendizaje, quiere decir entonces que representan una desviación de la normalidad y constituyen una necesidad educativa.

Por su parte Crozier (2001), explica que las diferencias individuales aluden a la diversidad más o menos notable que un alumno presenta al seguir el ritmo de aprendizaje de sus compañeros, cualquiera que sea el factor determinante en el proceso básico como memoria, metacognición, estrategias de aprendizaje, entre otros es determinante en la ventaja o retraso del mismo, puesto que detectan alteraciones en los diversos aspectos en el desarrollo de sus capacidades.

Luego de una revisión exhaustiva ante las definiciones antes planteadas los autores presentan similitud en que las diferencias individuales son aquellas originadas por diferentes factores lo cual implica para el individuo anormalidades o ventajas en su proceso de aprendizaje, por su parte Castejón (2009), señala que tienen que ver específicamente con motivaciones, estilos de aprendizaje y otros factores de personalidad, lo cual difiere de lo planteado por Woolfolk (2006), quien señala que se trata más bien de factores biológicos o por variaciones en las experiencias de aprendizaje.

Sin embargo, es Crozier (2001), que explica con mayor detalle cuáles son esas diferencias individuales de la metacognición, permitiéndole al mismo definirla como esa particularidad notoria del estudiante que refleja durante el proceso de aprendizaje entre sus compañeros lo cual le acarrea desventajas o beneficios ante el desarrollo de las diferentes asignaciones propuestas por el profesor.

\section{Orientación a la acción posterior al fracaso}

Para Crozier (2001), consiste en un estado de preocupación donde la persona no puede apartar de su mente los pensamientos irrelevantes para la tarea, vuelve una y 
otra vez sobre ellos siendo incapaz de concentrarse en el paso siguiente. Siempre que trata de concentrarse, surgen constantemente estos pensamientos, que ocupan el espacio de la memoria de trabajo e impiden un desarrollo satisfactorio de la tarea.

Por otra parte Woolfolk (2006), explica que algunas diferencias se deban al desarrollo donde explica que según estudios los niños pequeños tiene una memoria de trabajo limitada, pero que aumenta con la edad, sin embargo no resulta claro si tales diferencias son el resultado de cambios en la capacidad de la memoria o en la mejoría en el uso de las estrategias.

En palabras de Gento y Vivas (2003), una de las diferencias individuales es Ilamada juicios metacognitivos donde el aprendiz tiene que hacer juicios sobre sí mismo y sobre sus tareas. Los interrogantes sobre el esfuerzo, las expectativas, las dificultades y los resultados necesariamente incluyen interacciones sociales, disposiciones motivacionales y consecuencias de aprendizaje. Todos estos juicios implican, de alguna manera, una autoevaluación de la metacognición donde los juicios de las personas sobre los aspectos de la situación de aprendizaje preceden a sus actuaciones.

Los autores difieren en totalidad con sus definiciones sin embargo existe cierta similitud entre Crozier y Gento y Vivas (2003), puesto que a pesar de que este ultimo la denomina juicios metacognitivos coinciden en que el estudiante no puede dejar de hacer juicios sobre sí mismo siendo incapaz de concentrarse en el paso siguiente generando preguntas sobre el esfuerzo, las expectativas, las dificultades y los resultados. El autor identifica las diferencias individuales de una manera particular lo cual difiere de otros autores pero se adapta al contexto donde se encuentra desarrollada la investigación. Por su parte Woolfolk (2006), señala que se refiere a una memoria de trabajo limitada donde tal diferencia es el resultado de cambios en la capacidad de la memoria o en la mejoría en el uso de las estrategias.

\section{Orientación a la acción frente a dudas}

Inicialmente Crozier (2001), indica que en un estado de dudas, la capacidad de decidir algo se ve obstaculizada por la representación mental que la persona hace de los 
posibles resultados de la decisión, incluyendo los resultados remotos o improbables; resultando imposible prescindir de la información menos relevante y la persona se crea una representación excesivamente compleja de la situación. A pesar de esta evaluación de la información o quizá a causa de ella, no es capaz de actuar ni de comprometerse a una acción.

En cambio, Woolfolk (2006), explica que la diferencia individual que afecta a la memoria a largo plazo es el conocimiento. Además, señala que cuando los estudiantes poseen mayores conocimientos procesal y declarativo de dominio específico son más capaces tanto de aprender como de recordar material en ese dominio, donde el interés es otro factor relacionado al desarrollo de conocimientos y de su recuerdo.

Por su parte Gento y Vivas (2003), las denomina diferencias en las elecciones y acciones donde en el estudio de la metacognición aún queda por dilucidar cómo ésta dirige la conducta donde pareciera que tanto los juicios como los pensamientos metacognitivos guían las decisiones en momentos cruciales del aprendizaje escolar. Por su parte Crozier (2001), identifica las diferencias individuales de una manera particular lo cual difiere de otros autores, pero se adapta al contexto donde se encuentra desarrollada la investigación.

\section{Orientación a la acción frente a la volatilidad}

Según Crozier (2001), se parece a la preocupación porque está relacionada con dificultades para llevar las intenciones a la práctica y para mantener la atención sobre la tarea, aunque en este caso, la atención no se dirige hacia el yo, sino hacia otras tareas. Incluso cuando la tarea se realiza satisfactoriamente, al individuo le resulta difícil concentrarse en ella a expensas de otras actividades.

De acuerdo con Castejón (2009), explica que una de las diferencias individuales entre los alumnos se relaciona a la noción de la diversidad donde el concepto alude a cualquier diferencia notable que un alumno presenta al seguir el ritmo de aprendizaje de sus compañeros de edad, cualquiera que sea el factor determinante de la ventaja o el retraso, situándose principalmente en el conocimiento básico del que aprende, es decir 
que la diversidad de capacidades depende, no tanto de la capacidad cognitiva en sí misma sino de las habilidades cognitivas.

Para Castejón (2009), una de las diferencias individuales la denomina noción de necesidades educativas donde cada uno de los alumnos las presenta de manera particular, es decir cuando un estudiante afronta la situación de enseñanza-aprendizaje lo hace con arreglos a su estilo particular para aprender, a sus ideas, a sus centros de interés, a sus conocimientos previos, entre otros. En función de ello, los distintos alumnos avanzan en su aprendizaje con ritmos diferentes, con estrategias de aprendizaje distintas y con mayores o menores dificultades.

Crozier (2001), identifica las diferencias individuales de una manera particular lo cual difiere de otros autores, pero se adapta al contexto donde se encuentra desarrollada la investigación.

\section{MATRIZ METODOLÓGICA}

La Investigación se califica como descriptiva, debido a que la misma describirá el problema planteado a través del análisis de las variables objeto de estudio, describiendo la situación existente en la población a estudiar. Al respecto Sabino (2012), plantea que las investigaciones descriptivas radican en describir algunas características fundamentales de un conjunto homogéneo de fenómenos, utilizando criterios sistemáticos que permitan poner de manifiesto su estructura o comportamiento. De esta forma se puede obtener una información sistemática sobre las mismas. Es decir, miden o evalúan diversos aspectos, dimensiones o componentes del fenómeno a estudiar (Bavaresco, 2006). Tal como ocurrirá durante la investigación donde serán descritas las diferentes situaciones y características relacionadas con los estudiantes que fueron objeto de análisis.

Igualmente se categorizó como no experimental, de campo y transeccional. Por cuanto Hernández, Fernández y Baptista (2010) lo señalan como aquel en el cual no existe manipulación de variables por parte del investigador. Precisamente de campo argumentado por Tamayo y Tamayo (2009), establece los diseños de campo son 
cuando: "si queremos conocer algo sobre el comportamiento de las personas, lo mejor es preguntarles directamente a ellas". Al respecto, Chávez (2007) asevera que los estudios transeccionales son aquellos que miden una vez la variable; se miden los criterios de uno o más grupos de unidades en un momento dado, sin pretender evaluar la evolución de esas unidades.

La población para el desarrollo de la temática seleccionada se encontró conformada por un grupo de estudiantes de educación media de las instituciones educativas pertenecientes al 5to grado de educación media, sin discriminar entre géneros ni rendimiento estudiantil. En este orden de ideas, como población sujetos de estudio, se escogieron de forma intencional por conveniencia algunas secciones de las instituciones de la comuna de Nuñoa en la ciudad de Santiago, los cuales totalizaron 119 estudiantes, que utilizan las tecnologías de información para el desarrollo de las actividades académicas, ello permitió observar el nivel de presencia de las habilidades congnitivas.

Se aplicó una encuesta contentiva de 18 itemes con escalamiento tipo Likert, para la recolección de la información, durante el período entre abril y junio del 2018, la cual fue estructurada con cinco (5) opciones de respuestas: Siempre, casi siempre, a veces, casi nunca y nunca. Para determinar la fiabilidad del instrumento se aplicó una prueba piloto a 15 estudiantes seleccionados sin que formaran parte de población final, procediendo a calcular el coeficiente de confiabilidad mediante la fórmula de Alfa de Cronbach, la cual arrojó un índice de 0,93, determinado que el instrumento es confiable.

\section{RESULTADOS}

Los resultados de la aplicación del instrumento a la población objeto de estudio fue tabulado utilizando una tabla de doble entrada. En este sentido se evidencian los siguientes resultados obtenido en los estudiantes de educación media que fueron objeto de estudio.

Para la interpretación de los resultados se diseñó un baremo interpretativo que facilitó la búsqueda de categorías y la cual se muestra en la siguiente tabla: 
KOINONIA. Revista Arbitrada Interdisciplinaria de Ciencias de la Educación, Turismo, Ciencias Sociales y Económica, Ciencias del Agro y Mar y Ciencias Exactas y aplicadas. Año IV. Vol IV. N7. Enero - Junio 2019. Hecho el depósito de Ley: FA2016000010 ISSN: $2542-3088$

FUNDACIÓN KOINONIA (F.K). Santa Ana de Coro. Venezuela.

Natalie del Carmen Muñoz Morales; Nicolás Pablo Barrientos Oradini; Luis Araya Castillo; Jaime Reyes Saavedra

Cuadro 1. Categorización para el análisis de los resultados

\begin{tabular}{|c|c|c|}
\hline Escala de Valores & Alternativas & Categorías \\
\hline $3.26 \ldots \ldots \ldots . .4 .00$ & Siempre & Adecuada \\
\hline $2.51 \ldots \ldots \ldots \ldots .3 .25$ & Casi Siempre & $\begin{array}{c}\text { Medianamente } \\
\text { Adecuada }\end{array}$ \\
\hline $1.76 \ldots \ldots \ldots \ldots .5 .50$ & Casi Nunca & Poco Adecuada \\
\hline $1.00 \ldots \ldots \ldots \ldots .75$ & Nunca & Inadecuada \\
\hline
\end{tabular}

De esta forma y en base a los resultados obtenidos, se ubican las alternativas seleccionadas en la escala de valores seleccionada por el investigador, para indicar la categoría en la cual se sitúa cada uno de los indicadores que conforman las dimensiones que estructuran la variable objeto de estudio

La tabla 1, muestra la distribución de frecuencias de los indicadores de la dimensión Habilidades Metacognitivas. Con respecto al indicador Planeación, el 84\% de los encuestados respondieron que siempre organizan todas las actividades asignadas en clases, buscan con tiempo las asignaciones que les coloca su docente y siguen los pasos de forma ordenada para realizar las tareas. Mientras el 16\% respondió casi siempre.

Tabla 1. Habilidades Metacognitivas

\begin{tabular}{|c|c|c|c|c|c|c|}
\hline \multicolumn{7}{|c|}{ HABILIDADES METACOGNITIVAS } \\
\hline INDICADORES & \multicolumn{2}{|c|}{ Planeación } & \multicolumn{2}{|c|}{ Verificación } & \multicolumn{2}{|c|}{ Evaluación } \\
\hline Alternativas de respuestas & $\mathbf{F a}$ & $\%$ & $\mathrm{Fa}$ & $\%$ & $\mathbf{F a}$ & $\%$ \\
\hline Siempre & 100 & $84 \%$ & 82 & $69 \%$ & 91 & $76 \%$ \\
\hline Casi Siempre & 19 & $16 \%$ & 31 & $26 \%$ & 28 & $24 \%$ \\
\hline Casi Nunca & 0 & $0 \%$ & 6 & $5 \%$ & 0 & $0 \%$ \\
\hline Nunca & 0 & $0 \%$ & 0 & $0 \%$ & 0 & $0 \%$ \\
\hline Total & 119 & $100 \%$ & 119 & $100 \%$ & 119 & $100 \%$ \\
\hline Promedio & \multicolumn{2}{|c|}{3,55} & \multicolumn{2}{|c|}{3,33} & \multicolumn{2}{|c|}{3,61} \\
\hline Interpretación de la Media & \multicolumn{2}{|c|}{ Altamente adecuado } & \multicolumn{2}{|c|}{ Adecuado } & \multicolumn{2}{|c|}{$\begin{array}{l}\text { Altamente } \\
\text { adecuado }\end{array}$} \\
\hline
\end{tabular}


También se observa la media aritmética para este indicador con un valor de 3,55; el cuál se ubica en el rango de la categoría altamente adecuado del baremo. Esta tendencia se corresponde con los postulados teóricos de Woolfolk (2006), quien refiere que la planeación implica cuanto tiempo dedicar a una tarea, que estrategias utilizar, como empezar, que recursos reunir, que orden seguir, que revisar de forma superficial y a que ponerle mayor atención.

De igual manera en la tabla 3, se puede observar el indicador Verificación, como un componente de la dimensión Habilidades Metacognitivas. El resultado de la encuesta arrojó, que el $69 \%$ de los sujetos respondieron que siempre verifican antes de presentar un examen todo el material leído, también la fuente del material que descargan de Internet y el tiempo que utilizan para realizar un proyecto en clase. Mientras $26 \%$ respondió casi siempre y 5\% casi nunca.

De la misma forma se observa la media aritmética para con un valor de 3,33; el cuál se ubica en el rango de la categoría adecuado del baremo. Se infiere que coincide con los postulados teóricos de Woolfolk (2006), quien refiere que la verificación es la conciencia continua de preguntarse la manera en que se están haciendo las cosas, de tal manera que verificar en indagar si tiene sentido lo ejecutado, si se ha tomado el tiempo suficiente para realizarlo o si se ha estudiado lo necesario.

Es decir que es reafirmar que se está haciendo lo correcto, o reconstruir lo planeado para hacerlo de la manera correcta que le permita al estudiante concretar lo propuesto como pasar el examen con una buena calificación, realizar proyectos de investigación, asegurar su futuro de un modo correcto, entre otros.

Asimismo, se puede observar el indicador Evaluación, como un componente de las Habilidades Metacognitivas. El resultado de la encuesta arrojó, que el $76 \%$ de los sujetos respondieron que siempre al terminar una actividad es evaluado su desempeño, los resultados negativos son tomados en cuenta para mejorar y a partir de los resultados obtenidos son propuestos nuevos objetivos. Mientras $24 \%$ respondió casi siempre. 
De la misma forma se observa la media aritmética con un valor de 3,61; el cuál se ubica en el rango de la categoría altamente adecuado del baremo. Esta tendencia se corresponde con los postulados teóricos de Woolfolk (2006), quien señala que la evaluación consiste en hacer juicios acerca de los procesos y resultados tanto del pensamiento como del aprendizaje emitiéndose preguntas como sobre las estrategias implementadas, las mejoras a lo realizado, la continuidad del proyecto, el grado de satisfacción una vez culminado el modelo, la tarea, el plan, entre otros, para de esta manera evitar la intervención de los razonamientos espontáneos incorrectos en el aprendizaje.

En relación a las diferencias individuales de la metacognición en los estudiantes de 5to media, se obtuvo la siguiente información:

La tabla 2 presentada a continuación, muestra la distribución de frecuencias de las Diferencias Individuales Metacognitivas. Con respecto al indicador Orientación a la acción posterior al Fracaso, el $71 \%$ de los encuestados respondieron que siempre al enfrentar una nueva experiencia en clases temen equivocarse, así como presentando los resultados de una investigación realizada, les cuesta retomar el control; sin embargo, aprenden de los errores que cometen en clase. Mientras 29\% respondió casi siempre.

También se observa la media aritmética para este indicador con un valor de 3,41 ; el cuál se ubica en el rango de la categoría altamente adecuado del baremo. Esta tendencia se corresponde con los postulados teóricos de Crozier (2001), quien refiere que la orientación a la acción posterior al fracaso consiste en un estado de preocupación donde la persona no puede apartar de su mente los pensamientos irrelevantes para la tarea, vuelve una y otra vez sobre ellos siendo incapaz de concentrarse en el paso siguiente. Siempre que trata de concentrarse, surgen constantemente estos pensamientos, que ocupan el espacio de la memoria de trabajo e impiden un desarrollo satisfactorio de la tarea. 
KOINONIA. Revista Arbitrada Interdisciplinaria de Ciencias de la Educación, Turismo, Ciencias Sociales y Económica, Ciencias del Agro y Mar y Ciencias Exactas y aplicadas. Año IV. Vol IV. N7. Enero - Junio 2019. Hecho el depósito de Ley: FA2016000010 ISSN: $2542-3088$

FUNDACIÓN KOINONIA (F.K). Santa Ana de Coro. Venezuela.

Natalie del Carmen Muñoz Morales; Nicolás Pablo Barrientos Oradini; Luis Araya Castillo; Jaime Reyes Saavedra

Tabla 2. Diferencias individuales de la Metacognición

\begin{tabular}{|c|c|c|c|c|c|c|}
\hline \multicolumn{7}{|c|}{ DIFERENCIAS INDIVIDUALES DE LA METACOGNICIÓN } \\
\hline \multirow{2}{*}{$\begin{array}{c}\text { INDICADORES } \\
\text { Alternativas }\end{array}$} & \multicolumn{2}{|c|}{$\begin{array}{l}\text { orientación a la acción } \\
\text { posterior al fracaso }\end{array}$} & \multicolumn{2}{|c|}{$\begin{array}{l}\text { Orientación a la } \\
\text { acción frente a } \\
\text { dudas }\end{array}$} & \multicolumn{2}{|c|}{$\begin{array}{l}\text { Orientación a la acción } \\
\text { frente a volatilidad }\end{array}$} \\
\hline & $\mathbf{F a}$ & $\%$ & Fa & $\%$ & $\mathbf{F a}$ & $\%$ \\
\hline Siempre & 84 & $71 \%$ & 94 & $79 \%$ & 97 & $82 \%$ \\
\hline Casi Siempre & 35 & $29 \%$ & 25 & $21 \%$ & 22 & $18 \%$ \\
\hline Casi Nunca & 0 & $0 \%$ & 6 & $5 \%$ & 0 & $0 \%$ \\
\hline Nunca & 0 & $0 \%$ & 0 & $0 \%$ & 0 & $0 \%$ \\
\hline Total & 119 & $100 \%$ & 119 & $100 \%$ & 119 & $100 \%$ \\
\hline Promedio & \multicolumn{2}{|c|}{3,41} & \multicolumn{2}{|c|}{3,44} & \multicolumn{2}{|c|}{3,55} \\
\hline $\begin{array}{c}\text { Interpretación de la } \\
\text { Media }\end{array}$ & \multicolumn{2}{|c|}{ Altamente Adecuado } & \multicolumn{2}{|c|}{ Altamente Adecuado } & \multicolumn{2}{|c|}{ Altamente Adecuado } \\
\hline
\end{tabular}

De igual se puede observar el indicador Orientación a la Acción Frente a Dudas, como un componente de la dimensión Habilidades Metacognitivas. El resultado de la encuesta arrojó, que el $79 \%$ de los sujetos respondieron que siempre toman decisiones en un proyecto de clases a pesar de las dudas que se les presentan, asumiendo el compromiso para realizarlas. Mientras $21 \%$ respondió casi siempre.

Se observa la media aritmética para este indicador con un valor de 3,44; el cuál se ubica en el rango de la categoría altamente adecuado del baremo. Esta tendencia se corresponde con los postulados teóricos de Crozier (2001), quien indica que, en un estado de dudas, la capacidad de decidir algo se ve obstaculizada por la representación mental que la persona hace de los posibles resultados de la decisión, incluyendo los resultados remotos o improbables; resultando imposible prescindir de la información menos relevante y la persona se crea una representación excesivamente compleja de la situación. A pesar de esta evaluación de la información o quizá a causa de ella, no es capaz de actuar ni de comprometerse a una acción. 
En el mismo orden de ideas, se presenta el indicador Orientación a la Acción Frente a Volatilidad, como un componente de la dimensión Habilidades Metacognitivas. El resultado de la encuesta arrojó, que el $82 \%$ de los sujetos respondieron que siempre las posibles dificultades en la ejecución de un proyecto suelen preocuparse, al momento de realizar una tarea se preocupan más por las demás y la atención en otras actividades es mantenida mientras realizan una tarea. Por otro lado, 18\% respondió casi siempre.

Asimismo, también se puede observar la media aritmética para éste indicador con un valor de 3,55; el cuál se ubica en el rango de la categoría altamente adecuado del baremo. Esta tendencia se corresponde con los postulados teóricos de Crozier (2001), quien refiere que la orientación a la acción frente a volatilidad se parece a la preocupación porque está relacionada con dificultades para llevar las intenciones a la práctica y para mantener la atención sobre la tarea, aunque en este caso, la atención no se dirige hacia el yo, sino hacia otras tareas. Incluso cuando la tarea se realiza satisfactoriamente, al individuo le resulta difícil concentrarse en ella a expensas de otras actividades.

\section{CONCLUSIONES}

Una vez realizado el análisis y discusión de los resultados, se procede a formular las conclusiones del proceso investigativo:

En relación a las habilidades metacognitivas empleadas por los estudiantes de 5to grado de la Institución Educativa Técnico Industrial Antonio Prieto Sede Santa María; concluyéndose que la gran mayoría de los escolares de la institución en contexto, perciben como altamente adecuado las habilidades metacognitivas, a través de la planeación, verificación y evaluación. No obstante, un grupo significativo lo percibe como adecuado y una minoría poco significativa como medianamente adecuado.

En función a las diferencias individuales de la metacognición en los estudiantes de 5to medio de las instituciones que fueron objeto de estudio en la comuna de Nuñoa de la ciudad de Santiago; concluyéndose que la gran mayoría de los escolares de las instituciones en contexto, perciben como altamente adecuado las diferencias 
individuales de la metacognición, a través de la orientación a la acción posterior al fracaso, orientación a la acción frente a las dudas y orientación a la acción frente a la volatilidad. No obstante, un grupo significativo lo percibe como adecuado.

\section{Reflexiones finales}

En este aparte se establecen las recomendaciones que emanan de los hallazgos de la investigación, enfocados hacia aquellos resultados que en el puntaje obtenido son susceptibles de mejoras. En ese sentido, cabe destacar que en el contexto objeto de estudio, los estudiantes, perciben de forma significativa el comportamiento y tendencia de los elementos componentes del uso de dispositivos móviles en el desarrollo de capacidades metacognitivas; sin embargo, es pertinente acotar que los resultados muestran algunas situaciones que deben someterse a consideración, por lo que se establecen las siguientes recomendaciones:

Con respecto a las habilidades metacognitivas y con la finalidad de mantener la categoría altamente adecuado, se recomienda reforzar en los estudiantes la planeación; para ello deben incentivarlos a organizar todas las actividades asignadas en clases, buscar con tiempo las asignaciones que les coloca.

Estimular a los estudiantes a hacer verificación de todo el material leído antes de presentar un examen, así como la fuente del material que bajan de Internet y el tiempo que utilizan para realizar un proyecto en clase. De igual manera, al terminar cada actividad, evaluar el desempeño, y proponer nuevos objetivos partiendo del resultado obtenido.

En lo relativo a las diferencias individuales de la metacognición, y con la finalidad de mantener la categoría altamente adecuado, se recomienda reforzar en los estudiantes la orientación a la acción posterior al fracaso, estimulándolos a enfrentar las nuevas experiencias en clases sin temor a equivocarse, y ayudarlos retomar el control en las presentaciones de los resultados de una investigación, a través de la técnica de respiración y concentración. 
En cuanto a la orientación a la acción frente a dudas, se recomienda orientar a los alumnos a que tomen sus propias decisiones en los proyectos de clases independientemente de las dudas que se les presentan, a través del compromiso para su realización.

En cuanto a la orientación a la acción frente a volatilidad, se recomienda, orientar a los estudiantes a afrontar las posibles dificultades en la ejecución de un proyecto, a superar su preocupan por la realización de sus tareas. Para ello, se sugiere estimular la autoconfianza y la concentración en las actividades que realizan.

Adicionalmente, se recomienda poner en práctica estrategias, orientadas al uso de los dispositivos tecnológicos, con la finalidad de desarrollar las capacidades metacognitivas en los estudiantes analizados.

\section{REFERENCIAS CONSULTADAS}

1. Bavaresco, A. (2006). Proceso Metodológico en la Investigación. Cómo hacer un diseño de investigación. Venezuela: La Universidad del Zulia.

2. Caballero, E. (2010) citado por Blanco (2015).Tecnologías Móviles En el desempeño Escolar En La Educación Media. Venezuela: Universidad Rafael Urdaneta. Venezuela.

3. Castejón, J (2009). Unas bases psicológicas de la educación especial. Editorial: Club Universitario. Recuperado de: https://books.google.co.ve/books?id=1iYAQeyYr3sC\&dq. Consultado: 02/02/2018.

4. Chadwick (1985) citado por Blanco (2015). Capacidades Metacognitivas. Consideraciones Teóricas Acerca De La Metacognición. Disponible En www.utp.edu.co/chumanas/revistas/revistas/rev28/vargas.htm.

Consulta 04/04/2018.

5. Chávez, N. (2007). Introducción a la investigación educativa. Venezuela: Editorial Gráficas González.

6. Chávez, N (2009), Introducción a la Investigación Educativa. Venezuela:Editorial Ars. Gráfica.

7. Caballero, C.C., Abello, R., y Palacio, J. (2007). Relación del burnout y el rendimiento académico con la satisfacción frente a los estudios en estudiantes universitarios. Avances en Psicología Latinoamericana, 25(2), 98-111. 
8. Cabrera, P y Galán, E. (2002). Satisfacción escolar y Rendimiento académico. Revista de Psicodidáctica, 14, 86-98.

9. Casares y Siliceo (2014). Planeación de Vida y Carrera. 2da Edición. Talleres de imprenta Andina, México.

10. Crozier, R (2001). Diferencias individuales en el aprendizaje: personalidad $y$ rendimiento escolar. Editorial: Narcea Ediciones. Recuperado de: https://books.google.co.ve/books?id=OmN0WmptJ9AC\&dq.

11. Flavell, J. (1978). Capacidades Metacognitivas. Consideraciones Teóricas Acerca De La Metacognición. Recuperado de: www.utp.edu.co/chumanas/revistas/revistas/rev28/vargas.htm. Consulta $11 / 07 / 2018$.

12. Gento, S. y Vivas, M. (2003). El SEUE: un instrumento para conocer la satisfacción de los estudiantes universitarios con su educación. Acción Pedagógica, 12(2), 1627.

13. Hernán, C (2014). Aprendizaje Móvil o M-Learning. Recuperado de: http://mlearning2012.blogspot.com/p/ventajas-y-desventajas.html.

14. Hernández, R, Fernández. C. y Baptista, P. (2010). Metodología de la Investigación. 5ta edición. México DF, México: Editorial Mc Graw- Hill Interamericana S.A.

15. Necuzzi, C (2013). Estado del arte sobre el desarrollo cognitivo involucrado en los procesos de aprendizaje y enseñanza con integración de las TIC. Recuperado de: https://books.google.co.ve/books?id=KRCABAAAQBAJ\&pg=PA79\&dq.

16. Organización de las Naciones Unidas para la Educación, la Ciencia y la Cultura (UNESCO, 2012). Educación de calidad para todos y el Proyecto regional de Educación para América Latina y el Caribe (PRELAC). Recuperado de: http://portal.unesco.org/geography/es/ev.phpRL_ID=7464\&URL_DODO_ TOPIC\&URL_SECTION=201.html. Consulta 08/02/2018.

17. Organización de las Naciones Unidas para la Educación, la Ciencia y la Cultura UNESCO (2013). Aprendizaje Mediante Dispositivos Móviles: "No Podemos Seguir Viviendo en la era Predigital. Recuperado de: http://www.unesco.org/new/es/education/resources/onlinematerials/singleview/news/mobile_learning_we_cannot_continue_to_live_in_the_pre _digital_era. 
KOINONIA. Revista Arbitrada Interdisciplinaria de Ciencias de la Educación, Turismo, Ciencias Sociales y Económica, Ciencias del Agro y Mar y Ciencias Exactas y

18. Rodríguez (2012) citado por Lizardo (2014). Uso De Dispositivos en Aprendizaje De Los Estudiantes De Los Programas Nacionales De Formación. Trabajo Especial De Grado. Maestría En Informática Educativa. Maracaibo, Venezuela. Universidad Rafael Belloso Chacin.

19. Sabino, C. (2012). El Proceso de la Investigación. Una introducción Teórico Práctica. Caracas- Venezuela: Editorial Panapo.

20. Tamayo, y Tamayo, M. (2009). El Proceso de Investigación Científica. México DF, México: Tercera edición. Editorial Limusa.

21. Unión Internacional de Telecomunicaciones (2009). Perfiles Estadísticos de la Sociedad de la Información 2009. Región América. Documento en línea. Recuperado de: https://www.itu.int/dms_pub/itu-d/opb/ind/D-IND-RPM.AM-2009E09-R1-PDF-S.pdf.

22. Woolfolk, A (2006). Psicología Educativa. Editorial: Pearson Education. Recuperado de: https://books.google.co.ve/books?id=PmAHE32RuOsC\&dq.

(C2019 por los autores. Este artículo es de acceso abierto y distribuido según los términos y condiciones de la licencia Creative Commons Attribution (http://creativecommons.org/licenses/by/4.0/). 